Lexis Vol. XLIII (1) 2019: 221-232

\title{
Los Viajes de Riva-Agüero
}

Jorge Wiesse Rebagliati

Universidad del Pacífico

\section{RESUMEN}

A propósito de Viajes de José de la Riva-Agüero (1885-1944), se aprecian algunos rasgos textuales relevantes: la construcción de la persona narrativa, la descripción de los paisajes y la identidad ideal entre la Castilla española y la Sierra peruana. Se discuten, también, las semejanzas y las diferencias entre Viajes y Paisajes peruanos (1955), el gran libro de viajes de Riva-Agüero.

Palabras clave: Libros de viajes, José de la Riva-Agüero, persona narrativa, siglo XX, descripciones de paisajes

\section{ABSTRACT}

Regarding José de la Riva-Agüero's Viajes, some textual traits are pinpointed: the construction of a narrative persona, the description of landscapes and the ideal identity between Spanish Castille and the Peruvian Sierra. Similarities and differences between Viajes and Paisajes peruanos [Peruvian Landscapes], Riva-Agüero's major travel book, are also discussed.

Keywords: Travel books, José de la Riva-Agüero, narrative persona, Twentieth Century, descriptions of landscapes 
Si bien algunos de los textos incluidos en este volumen de las Obras completas de José de la Riva-Agüero se refieren al espacio subcontinental sudamericano, la mayoría describe otros (Norteamérica, el Caribe, Europa, el Extremo Oriente, el Medio Oriente, África). Se trata casi de una singularidad. Son más frecuentes los testimonios de viajeros extranjeros sobre el Perú (Squier, Raimondi, Sztolcman) que los testimonios de peruanos sobre países que no son el suyo. Como lo señala Margarita Guerra Martinière, editora de este volumen, pocos son los relatos de viaje "[...] de peruanos que han dejado testimonios sobre el Viejo Mundo o sobre Estados Unidos, como fue José Arnaldo Márquez” (XX), o como lo fue también el historiador Carlos Wiesse Portocarrero, con textos sobre sus experiencias europeas a finales del siglo XIX y principios del XX (Recuerdos de un viaje en Suiza, En ciudad ajena y en tierras propias).

En nueve capítulos y un anexo, Margarita Guerra ordena cronológicamente el variado material que guarda el Archivo Histórico Riva-Agüero. Los viajes consignados en cuadernos y libretas son los siguientes: a Bolivia (del 1 al 13 de mayo de 1912); a Puno (del 13 al 15 de mayo de 1912; el 15 llega al Cuzco: Paisajes peruanos se inicia con la salida del Cuzco el 1 de junio de 1912); a Europa, en vísperas de la Gran Guerra (1914-1918), vía Jamaica y Cuba (a partir del 10 de julio de 1913; solo hay registro hasta el 21 de setiembre de 1913, cuando Riva-Agüero se disponía a viajar de Paray-le-Monial a París); a Europa (España, Italia, Bélgica, aunque probablemente visitó otros países), en el tiempo de Entreguerras, autoexiliado durante el gobierno de Augusto B. Leguía (de fines de 1919 a pasado setiembre de 1930, probablemente hacia fines de año: existe testimonio del viaje a Pisco en enero de 1931, el mismo año en que se desempeñó como alcalde de Lima, cargo que ejerció hasta 1932); a México, los Estados Unidos, Japón, China, Manchuria, Corea, Egipto, Marruecos, España, Italia, Suiza, Portugal, Brasil, Argentina (de septiembre de 1938 a después de agosto de 1940, última fecha consignada). El anexo incluye varios viajes a Pisco (enero de 1931, 30 de mayo a 2 de junio de 1936, 6 a 7 de marzo de 1938) y 
uno a Cañete (marzo de 1935). A pesar de lo prolijo del material, no es posible reproducir totalmente la imagen del Riva-Agüero viajero. Del texto se deducen viajes no registrados. Por ejemplo, en la entrada del 2 de julio de 1939, Riva-Agüero escribió que era la novena vez que veía la fuente Gaia y la torre del Mangia en la plaza de Siena (407-408). Sin embargo, es la primera vez que el lector se entera de que el historiador peruano había viajado a Siena.

Como ya se ha señalado - lo han hecho Jorge Armando Guevara, en la Presentación; y la responsable de este tomo XXVII de las Obras completas de José de la Riva-Agüero, Dra. Margarita Guerra-, nos enfrentamos aquí a una variedad de páginas, si bien no en busca de autor (la voz autoral de don José es rotunda, clara, a veces arbitraria), sí a un conjunto de textos cuya unidad ideal -el relato de viajes - no se cumple cabalmente en ninguno. Aunque con grados, se reconocen efectivamente los tres binomios que identifica Luis Alburquerque (recordado por Margarita Guerra en su introducción) como definitorios del género (factual/ ficcional, objetivo/ subjetivo, y descriptivo/ narrativo): se trata de textos factuales (de personas, lugares, monumentos y objetos reales es de aquello de lo que se habla), descriptivos (se describen, en efecto, esas realidades, con predominio de este modo sobre el narrativo) y subjetivos (pues opiniones, juicios y prejuicios, filias y fobias se expresan casi sin filtro alguno). Como es típico de los relatos de viaje, la narración es elemental, dictada por el itinerario. Sin embargo, su condición de nota, de apunte, de esbozo no permite en ninguno de los textos de los Viajes rivagüerinos una cristalización completa de los rasgos genéricos.

A diferencia de Paisajes permanos, lo descriptivo, que en el libro que relata el viaje de 1912 es un aspecto trabajado con refinamiento de orfebre verbal y plástico, en Viajes no se presenta sino como esbozos. No es raro que se reduzca a topónimos o a series de topónimos cuya expansión temática agrupa actividades ya casi tópicas como misas, cenas, reuniones con personalidades de la aristocracia, de la política o de la cultura, visitas al médico, redacción de cartas, gestiones bancarias etc. En una cultura de lo público como 
la nuestra actual, donde las intimidades se ventilan en las redes sociales, no llama la atención la consignación de lo doméstico, de las funciones corporales o de las enfermedades, pero en el mundo de Riva-Agüero lo público y lo privado se distinguían más, y se tiene a veces la impresión de estar asistiendo al espectáculo de una interioridad al que no se había sido invitado. No debe olvidarse nunca de que se está ante textos íntimos que no fueron preparados para la publicación por su autor y que Riva-Agüero, sobre todo en sus opiniones acerca de personas o de coyunturas políticas, quizás habría reformulado lo que aparentemente surgió como impresión de un instante o al calor de una polémica.

¿Cómo leer estos textos que, a diferencia de los recogidos en Paisajes peruanos, don José nunca pensó publicar? Pienso que proponiendo un ejercicio de lectura que continúe el de Margarita Guerra, y el de Andrés Llaury y Alonso Espinoza, pues, en verdad, no podemos considerar aún el trabajo hermenéutico iniciado por ellos como pleno, cabal o terminado. En este sentido, me gustaría centrarme en dos aspectos del relato de viajes que creo que podrían prolongar la labor esforzada y meritoria de Guerra, Llaury y Espinoza. Me refiero a la construcción de la persona narrativa de Viajes, o sea, al Riva-Agüero histórico - lo transcrito por ellos sería valioso como insumo para una biografía intelectual de don José-, y al esbozo más desarrollado de los que se presentan en estos materiales (aunque pueden reconocerse también en ellos fragmentos de artículos o de conferencias): me refiero a las descripciones.

En Viajes, puede encontrarse al narrador que no solo no teme juzgar, sino que lo hace frecuentemente, a veces modificando impresiones recogidas con anterioridad de un libro leído previamente o de un viaje previo. Y juzga desde la autoridad que le otorga su enorme erudición, pero también desde sus prejuicios —o sus posiciones - políticos, de casta y de raza. Riva-Agüero cree que el comunismo bolchevique (con el concurso enérgico o flébil del sionismo y la masonería) destruirá la civilización occidental; cree que la aristocracia tiene el deber de regir los destinos de la sociedad y cree que España y la raza latina han contribuido con ideales más 
elevados a la civilización occidental que Inglaterra y la raza sajona. Es nacionalista y cree que el Perú debe estar a la altura del destino que su historia y su tradición cultural le señalan. Se entienden en este contexto tanto sus entusiasmos por la Italia fascista, el Japón imperialista y la España nacionalista como sus críticas al ambiente intelectual norteamericano (estadounidense y mejicano) $-\mathrm{y}$ en general a la puerilidad de la cultura de masas (léase Hollywood) y a la política dictada por el gran capital (léase Nueva York). Las opiniones sobre Alfonso Reyes (a quien aprecia, por otro lado), Pedro Henríquez Ureña, Franz Tamayo, Jacques Maritain, Georges Bernanos, Miguel de Unamuno y José Ortega y Gasset, por ejemplo, están teñidas - como todas- por este "eje" ideológico. Lo que no excluye matices y prevenciones: considera a Hitler un verdadero peligro —el juicio es de 1939- y rechaza tanto su política antijudía como sus permanentes roces con la Iglesia Católica. Por ejemplo, parece seriamente preocupado por la situación internacional en la entrada del cuaderno de apuntes del 14 de setiembre de 1938:

Pero en este mundo feo, barbarizado y ominoso de la post-guerra, la nueva guerra inexplicable que se anuncia, nos halla con los ideales confusos, y entreveradas esperanzas. Si triunfan Rusia, Francia y sus amigos, será por el resultado de la vez pasada [se refiere a la Gran Guerra, a la Primera Guerra Mundial]. Será la plebeyización de todo, política, arte y pensamiento; el reino del mal y la pequeñez anímica. Pero si triunfan Alemania nazista y sus aliados, será el despotismo temible, la intolerancia contra las otras razas reputadas inferiores, la lid insensata y suicida contra el catolicismo; para Italia, en medio de su victoria subalterna, las más efectivas amenazas, la situación de podestá del nuevo Kaiser, harto más duro que el pobre Guillermo de Hohenzollern, y para nuestros países sudamericanos, el amago de contrapuestas ambiciones colonizadoras. Perspectiva horrorosa. Apenas veo posibilidad de que el conflicto se evite (172).

Y reacciona con horror aún más sentido ante las noticias de la Noche de los Cristales Rotos, la Kristallnacht, la orgía de destrucción causada por las tropas de la S.S. y sufrida por casas, negocios y sinagogas judíos el 9 y el 10 de noviembre de 1938. En la entrada del 
jueves 10 al viernes 11 de noviembre de 1938, mientras está en San Francisco, Riva- Agüero escribe:

Llegan en estos días noticias lastimosas de Alemania. Las persecuciones contra los judíos reviven las más tristes escenas medioevales; y los católicos siguen vejados en sus prelados y amenazadísimos por la canallesca audacia de los racistas, que no alcanzan a comprender que sin el auxilio de las religiones, y en particular de la católica, la guerra contra el comunismo pierde su alma, su dirección, su más seguro estandarte (224).

Por otro lado, sin dejar de criticar la injerencia de los Estados Unidos en la política continental mediante la doctrina del panamericanismo, reconoce la bonhomía del pueblo estadounidense, que merece el bienestar económico del que goza — aunque Riva-Agüero no deja de hacer notar los estragos de la depresión- por estar compuesto de individuos "laboriosos, sensatos y en (su) mayoría, buenos” (201).

Puede ser útil para el historiador político o de la cultura revisar el texto de Viajes en busca de testimonios — siempre claros- de José de la Riva-Agüero sobre personajes que forjaron la historia contemporánea, con quien el polígrafo limeño trató directamente y fungió, motu proprio, de embajador de los intereses peruanos, americanos, latinos, católicos y occidentales, tal como él los concebía. Por citar a algunos de estos personajes: el Papa Pío XI (Achille Ratti), (159); el Almirante Yamamoto (Shinijiro, cabeza de los católicos japoneses y caballero de la Orden de Malta, y no Isoruku, el jefe de la flota imperial responsable del ataque a Pearl Harbor), (226); el rey Alfonso XIII, con quien almorzó (395); el general Francisco Franco (465) y varios jerarcas nacionalistas españoles, como los ministros Ramón Serrano Suñer y Rafael Sánchez Mazas (433), y el general José Moscardó (455). Con don Ramón Menéndez Pidal trató de la ayuda que podía brindarle don José al historiador Claudio Sánchez Albornoz (459), ayuda que nunca se cristalizó.

Los diplomáticos peruanos fueron interlocutores privilegiados y fuentes de noticias de un país cuya política seguía con dolido 
interés: Ricardo Rivera Schreiber (251), Víctor Andrés Belaunde (381), Raúl Porras Barrenechea (446), Ricardo Vegas García (500). Puede ser interesante registrar las impresiones de Riva-Agüero respecto de su frustrada postulación a la presidencia del Perú en 1939 (entrada del sábado 11 de noviembre de 1939):

De los periódicos que me han llegado, por José Pardo, resulta que en carta auténtica de Flores [Luis A. Flores, líder de la Unión Revolucionaria, partido de impronta fascista que apoyó a Sánchez Cerro] se dice que Beltrán [Pedro Beltrán Espantoso, líder del Partido Nacional Agrario y luego director de La Prensa a partir de 1947] le inspira mucha más confianza que yo. Recomendó la unión de las derechas sobre una alianza con El Comercio, el grupo de Villarán [Manuel Vicente Villarán], el mío y el de Beltrán; pero insiste en que para candidato los mejores según él eran Beltrán o [José] Quesada [Larrea]. El Comercio estuvo un tiempo entusiasta por mi candidatura, mas luego declaró que por ninguno lucharía abiertamente. La fecha de la carta de Flores es el 8 de julio de este año 39, en Valparaíso. La terna de El Comercio, según carta de José Quesada a Flores, ponía en primer lugar mi nombre, luego el del doctor Ernesto Delgado [el fundador de la Clínica Delgado], y en tercer término Quesada.

Se ve que Flores en su necedad, cree menos plutocrático y civilista a Beltrán [...] Beltrán está ausente como yo. Quizá se imaginaba Flores que proporcionaría dinero; su grupo se ha hecho hoy pradista. El Banco Italiano ofreció a Quesada los recursos que a mí, conocido italianófilo, no me ofreció en igual coyuntura. Tienen lo que merecen. Estoy más libre, tranquilo, sin haber llevado una derrota electoral. Hoy lunes el periódico madrileño Informaciones anuncia el triunfo de [Manuel] Prado [Ugarteche]. Quesada, por cartas publicadas en Lima, estuvo en tratos con [Víctor Raúl] Haya [de la Torre, líder del APRA] y con otros apristas; conversaciones indirectas y clandestinas. Para eso yo no sirvo (447-448).

Para Riva-Agüero (aún heredero de Taine), cultura y raza (aunque Taine hable más bien de carácter) son categorías que se superponen o se mezclan. Así, por ejemplo, puede sostener que "El mestizaje franco-moro explica toda la Edad Media española" 
(142). Las caracterizaciones étnicas o las explicaciones que acuden a la historia comparada (a las que se refiere Jorge Armando Guevara en su Presentación, p. X) son elaboraciones y desarrollos de este principio hermenéutico fundante, que es el de la fusión de categorías culturales y raciales. Los vascos, según don José, son helvéticos, más europeos que los castellanos (69): "Su misma creación religiosa, el jesuitismo, fue una componenda con el siglo, algo refinado, sutil, medio italiano. Son hermanos del alma de los suizos en todo." (69) En cambio, "Andalucía es una Italia menor, una Sicilia occidental, con más sello árabe.” (59)

Es indispensable que aparezca el tercer componente de la tríada de Taine: el paisaje. Aquí sorprende que Riva-Agüero, ciudadano del mundo, recurra permanentemente como término de comparación al Perú. Un primer ejemplo, de muchos, la descripción del paisaje de Badajoz:

El Guadiana ancho, con pedrones, medio seco, reproduce la imagen de un río peruano. Las colinas violetas del sur parecen los médanos de un valle costeño. Los labriegos, cetrinos, negrísimos, con el pelo rizoso, parecen zambos o cholos; con mantas de policromía india y monteras como en el Cuzco. (69) [mis cursivas].

Otro, más lejano en el tiempo y en el espacio, Egipto:

El campo egipcio se parece mucho al costeño del Perú, sobre todo al de Cañete, Pisco e Ica: desiertos de arena, tablazos áridos, con médanos y plantas de la aridez, cactos, una que otra palmera; y de golpe, adonde llegan los canales o acequias, el campo del algodón, los densos palmerales en la llanura, potreros de alfalfa, sauces y eucaliptos ralos y canchas de adobes, con azoteas idénticas a las nuestras, o casas de cine, estaciones con jardinillos minúsculos, como en La Joya y Vítor en Arequipa, adelfas floridas, recuas de burros y mulas, gente morena, mujeres gordas de ojos profundos y muchos negros; y hasta ahora, en invierno, el cielo cubierto de una niebla blanquezca, grisácea y con garúas limeñas, que de vez en cuando interrumpen este sol africano. En las ciudades, balcones con celosías de rejillas, mujeres con mantas negras y con rebozos como el antiguo limeño. Camellos como llamas grandes (321). 
La remisión al término de comparación prínceps, es decir, al Perú, no se reduce al paisaje, como acabamos de apreciar — consúltense también las páginas 29, 100, 309-. No es difícil encontrar ejemplos que ilustren este modo retórico que quizás sea testimonio de una peruanidad raigal. De la catedral de Pamplona dice que luce una "Fachada neoclásica con dos torres a la limeña" (120). En Santa Bárbara, California, come "tamales distintos y más suaves que los de mi tierra” (187). A veces, en la comparación, sufre el término por comparar. De los templos de Nara, en el Japón, afirma que "Al lado de nuestras catedrales y basílicas, esto es ramplón y paupérrimo" (267). Finalmente, y ello ofrece indicios de cuál podría ser el paradigma vital de Riva-Agüero, además de referirse a fenómenos $\mathrm{u}$ objetos peruanos, usa quechuismos y hasta términos quechuas como descriptores. Al aludir, por ejemplo, a un rasgo dialectal del italiano, la gorgia toscana, Riva-Agüero acude tanto al español como al quechua: "Habla dura: $C$ aspirada, casi como las jotas castellanas o la $c$ aspirada del quechua cuzqueño." (164). Este modo expresivo (y quizás espiritual) se aprecia, sobre todo, al describir el desierto de Palestina:

Al fin, el desierto, con gargantas secas (buaycos como en el Perú).

Se llega por estos portachuelos o llocllas (como dicen en mi tierra) a un lugar que está al nivel del Mediterráneo; y se sigue bajando. Comienza la llanura verdaderamente desértica; ya en esto es como nuestra costa, en vez que el primer trecho hasta acá es como nuestra puna de icho (352) [mis cursivas].

Para terminar, dos observaciones, que pueden aportar perspectiva a Paisajes peruanos, un libro que, a la vez, está muy cerca y muy lejos de los textos de Viajes. La primera se relaciona con la pertinencia editorial (primero, de Riva-Agüero, y, luego, de Raúl Porras y de César Pacheco) de no haber incluido a Bolivia en el plan de Paisajes, a pesar de que fue parte del itinerario del viaje de 1912. Por lo que podemos apreciar a través de las notas publicadas en Viajes, las referencias al paisaje boliviano son mínimas. Sin cumplir misión oficial, Riva-Agüero es tratado como un estadista peruano 
en Bolivia y discurre y opina sobre política continental —especialmente sobre las relaciones con Ecuador y con Colombia- como si estuviera ejerciendo efectivamente algún cargo público. En este subconjunto textual, el paisaje aparece brevemente y solo a partir del regreso al Perú, en Puno, aunque no con la cualidad sinfónica de los lienzos plásticos de Paisajes peruanos.

La segunda observación apunta a la identidad, en la experiencia vital de Riva-Agüero, de la sierra peruana y de Castilla. En la entrada del 17 de diciembre de 1920, en el viaje de Logroño a Madrid, concretamente en Pinares, después de Valladolid, Riva-Agüero confiesa: "Leo en el camino los versos de Campos de Castilla de Antonio Machado. Repite bastante sus sensaciones y expresiones, pero da muy bien el tono íntimo de este paisaje” (116). Antes, el 6 de mayo del mismo año, en el curso de una excursión a Toledo, don José había manifestado: “A mí Castilla me atrae por todo lo que hay en mí de consciente y de inconsciente, por mi razón y mis fuerzas oscuras” (47-48). Y remata así, ya el 7 de mayo:

He recorrido una de las regiones más típicas de España, en primavera radiante y calurosa. La idea literaria del país no es inexacta [Riva-Agüero puede estar refiriéndose a las reflexiones de los escritores de la Generación del 98 -o a las de alguno de la del 14]. La observación la confirma; y el parecido con la sierra peruana no es una ilusión [,] solo que el Perú es mucho más elevado y fragoso. Pero los canchales, las dehesas roquizas son como nuestras punas, con mayor arbolado es cierto, pero con igual majestad decorosa y devastada (48).

Se aprecia en el texto anterior, creo, la expresión de la intuición de una identidad profunda, que confirma a Riva-Agüero en su intento (hacia 1920, ya cristalizado parcialmente) de caracterizar al Perú a partir de la descripción de su paisaje (sobre todo del de su sierra), tal como hicieron los escritores del 98 con Castilla.

El esfuerzo de la transcripción por Alonso Espinoza de un material tan vasto y complejo (Riva-Agüero sobrescribía; algunas hojas de las libretas se encuentran dañadas por los ácidos de la tinta) se aprecia a pesar de algunos errores significativos como la escritura 
de "Vancluse" (134) por "Vaucluse", la comarca provenzal donde vivió Laura de Noves, la amada de Francesco Petrarca. La ortografía correcta del término está consignada en el índice toponímico. Del mismo modo, "San Miguel de Lino" (149-151) es una transcripción incorrecta de "San Miguel de Lillo", la iglesita prerrománica asturiana cercana a Oviedo. No es difícil confundir en la transcripción paleográfica la ene con la elle, sin embargo, tanto esta como Santa María del Naranco (150) son monumentos famosos del arte medieval asturiano y no oscuras capillas provinciales como para no registrarlos con la ortografía correcta. Tampoco es monumento menor la iglesia de Ognisanti ("Todos los Santos") de Florencia, mal escrita como "O Grisanti" (164). Los autores del políptico de Gante, La adoración del cordero pascual, son los hermanos Van Eyck (y no los "Vanlyck") (128); y el fresco de Rafael en la estancia de la Segnatura del Vaticano se titula La misa o El milagro de Bolsena y no El milagro de Brisena (392). "Sánchez Mojas" (449) es Rafael Sánchez Mazas, fundador de la Falange y ministro de Franco, sobreviviente de un fusilamiento a finales de la guerra civil española por gracia de un soldado republicano, historia narrada con solvencia por Javier Cercas en Soldados de Salamina.

En otros casos, para facilidad de la lectura, bien pudo haberse completado lo que aparece ilegible, pero aun así abierto a la conjetura. Al dar cuenta del camino que va de Santander a Covadonga, se transcribe lo siguiente: "Rincones de verdura entre el mar y los Picos de (ilegible) [...]" (148). Por la ruta, el topónimo no puede ser otro que los Picos de Europa. Ocurre también que con la indicación de "(sic) [= 'así]", con que se señala aquello que aparece curioso o incorrecto, se identifican expresiones perfectamente válidas, como "rascacielos ninivitas (sic)" (181) por los edificios de las ciudades estadounidenses. Son ninivitas porque, a semejanza de los zigurat de la ciudad mesopotámica de Nínive, se yerguen altos como queriendo rozar el cielo. 


\section{Referencias bibliográficas}

Riva-AgÜERo, José de la

1969 Paisajes peruanos. Obras completas de José de la Riva-Agüero, IX. Estudio preliminar de Raúl Porras Barrenechea. Lima: Pontificia Universidad Católica del Perú.

2018 Viajes. Obras completas de José de la Riva-Agüero, XXVII. Lima: Pontificia Universidad Católica del Perú. Instituto Riva-Agüero.

Recepción: 27/12/2018

Aceptación: 18/03/2019 